\title{
Evaluation of patients with clinically detected recurrence of rectal carcinoma: Current practice patterns of colorectal surgeons
}

\author{
ANKUR SANGOI ${ }^{1}$, UDAY PATEL ${ }^{3}$, KENICHI ODE ${ }^{2}$, RICCARDO AUDISIO $^{3}$, \\ KATHERINE S. VIRGO ${ }^{4}$ and FRANK E. JOHNSON ${ }^{5}$ \\ ${ }^{1}$ Department of Pathology, Stanford University Medical Center, Stanford, CA 94305, USA; ${ }^{2}$ Education Centre, \\ Royal Lancaster Infirmary, Lancaster LA1 4RR; ${ }^{3}$ Department of Surgery, Whiston Hospital - Prescot, \\ Merseyside L35 5DR, UK; ${ }^{4}$ American Cancer Society, Atlanta, GA 30303-1002; \\ ${ }^{5}$ Department of Surgery, Veterans Affairs Medical Center, St. Louis, MO 63106, USA
}

Received August 8, 2009; Accepted October 23, 2009

DOI: 10.3892/ol_00000063

\begin{abstract}
The optimal evaluation of patients with clinically suspected recurrence of rectal carcinoma following initial treatment has yet to be determined. We documented the intensity of the extent-of-disease workup conducted by colorectal surgeons when their patients with rectal carcinoma develop clinical evidence of metastases. A custom-designed questionnaire was mailed to all 1,795 members of the American Society of Colon and Rectal Surgeons. Subjects were asked which laboratory tests and imaging studies they would order for one of their own generally healthy patients with a suspicious abnormality found during surveillance testing. The tests most frequently recommended were computed tomography and serum carcinoembryonic antigen level. Few tests were recommended by $>90 \%$ of respondents. There is no consensus among experts in this common situation.
\end{abstract}

\section{Introduction}

Rectal carcinoma is common. The economic burden is substantial. Over 40,000 patients are diagnosed with this disease in the US each year, and approximately $65 \%$ are alive five years later (1). A sizeable minority of patients who develop recurrence following curative intent treatment are candidates for salvage treatment with curative intent. For others, effective chemotherapy is now available.

There are no evidence-based guidelines on which to base the evaluation of the extent of disease in a patient with rectal cancer who presents with clinical evidence of recurrence following curative-intent initial treatment. In order to

Correspondence to: Dr Frank E. Johnson, Department of Surgery, Saint Louis University Hospital, 3635 Vista Ave. at Grand Blvd., St. Louis, MO 63110, USA

E-mail: frank.johnson1@va.gov

Key words: rectal carcinoma quantify the intensity of rectal cancer patient workup under such circumstances, we recently surveyed members of the American Society of Colon and Rectal Surgeons (2). This is a large group of highly credentialed, well-recognized experts whose collective experience is enormous. This survey provided an opportunity to investigate the current strategies used by this group of experts and to document variation in diagnostic testing intensity.

Variation in clinical practice suggests that scarce health care resources are being overutilized, underutilized or misutilized (3). The Institute of Medicine considers this to be a serious problem in medical practice (4). Among the many sources of variation are physician age (5), geographic location of the physician (6-8), and (in the USA) health maintenance organization penetration rate. There are obvious reasons why these factors may influence clinical decision making. If older surgeons have not modified their clinical practice to accommodate new diagnostic and therapeutic modalities, one would anticipate that older surgeons and their younger counterparts would utilize tests differently when confronted with a rectal cancer patient with clinical evidence of recurrence. Similarly, if a surgeon practices in a region of the country without ready access to a particular sophisticated technique, one would anticipate that such a technique would be utilized sparingly. There may also be differences in testing intensity based on the health maintenance organization penetration rate because of restrictions in decision making by the health maintenance organization. Another likely source of variability is the clinical scenario leading to suspicion of recurrence (9). Serum carcinoembryonic antigen elevation in an asymptomatic patient may well be evaluated differently than evidence of lung metastases. We, therefore, analyzed the results of our previous survey to determine whether a patient with T3N0M0 rectal carcinoma who received curative-intent treatment and who presented with a carcinoembryonic antigen elevation detected during scheduled surveillance should be evaluated differently than a similar patient presenting with evidence on a surveillance chest X-ray of potentially resectable lung metastases. Our objective was to quantify variability among strategies and to assess whether physician age, US Census 
Region, health maintenance organization penetration rate, or type of suspected recurrence was able to account for the variability observed.

\section{Materials and methods}

The design of this study has been described in detail (2). In summary, we mailed a survey to all 1,795 active members of the American Society of Colon and Rectal Surgeons. The survey consisted of a cover letter explaining the purpose and nature of the investigation as well as the survey instrument itself. The full text is available on the Internet (http://surgery. slu.edu/survey/surgerysurvey.pdf). The first part of the instrument inquired whether the surgeon actually performed potentially curative resections of rectal cancer and whether he or she provided long-term postoperative follow-up for such patients. Only those surgeons who both operate on rectal cancer patients and follow up them postoperatively were asked to complete the questionnaire.

Respondents were asked to provide demographic information and to select all tests or procedures that they would perform on their initial workup in two clinical case vignettes. A review of consensus conference (10) and other pertinent literature (11) allowed us to compile a list of all testing modalities considered clinically useful in the postoperative setting; this was presented in menu form in the survey. The first scenario described a generally healthy patient who feels well overall, returns for scheduled follow-up two years after potentially curative low anterior resection of a T3NOM0 adenocarcinoma of the rectum (without adjuvant therapy), and who now has a serum carcinoembryonic antigen level of $8 \mathrm{ng} / \mathrm{dl}$ (normal, 1-5 ng/dl). The second scenario described a generally healthy patient who feels well overall, returns for scheduled follow-up two years after potentially curative low anterior resection of a T3NOM0 adenocarcinoma of the rectum (without adjuvant therapy), and who now has a serum carcinoembryonic antigen level of $1.4 \mathrm{ng} / \mathrm{dl}$ as well as a chest X-ray showing four $0.5-$ to $1.0-\mathrm{cm}$ radiodensities in both lung fields suggestive of metastases. In both scenarios, physical examination is not noteworthy.

On receipt of the completed surveys, the data were entered into a computerized database (Statistical Package for the Social Sciences). Frequency tables were generated for each modality. The $\chi^{2}$ analysis (or Fisher's test, as appropriate) was used to compare test use vs. non-use by surgeon age $(\leq 39$, 40-49, 50-59 and $\geq 60$ years), US Census Region (Northeast, South, Midwest and West, plus a separate category for non-US members of the American Society of Colon and Rectal Surgeons), health maintenance organization penetration rate ( $\leq 30$ and $>30 \%$ ), and clinical scenario for all modalities. Test use was reported as dichotomous ( 0 , no; 1 , yes). Statistical significance was set at $\mathrm{p}<0.05$.

\section{Results}

Of the 1,795 members of the American Society of Colon and Rectal Surgeons surveyed, 566 (32\%) completed the survey. Twenty-six of the $566(5 \%)$ who returned the survey stated that they typically do not provide long-term follow-up care. An additional 60 of the $566(11 \%)$ no longer perform rectal cancer surgery but were not listed as retired. One hundred and thirty-one of the $566(23 \%)$ did not follow the instructions properly and 2 of the $566(0.4 \%)$ respondents refused to complete the survey. These four subsets were considered nonevaluable $(n=219)$ and no further evaluation was performed. The remaining 347 of the $566(61 \%)$ respondents filled out the survey correctly, they performed rectal cancer surgery and followed up their patients postoperatively. These respondents were considered evaluable. Evaluable respondents were generally similar to non-evaluable respondents, suggesting that the survey results are reasonably representative of members of the American Society of Colon and Rectal Surgeons overall. Of the 347 evaluable respondents, $53(15 \%)$ were $\leq 39$ years of age, $131(38 \%)$ were $40-49,133(38 \%)$ were $50-59$ and $30(9 \%)$ were $\geq 60$.

The diagnostic modalities selected by $>50 \%$ of the evaluable respondents in assessing the patient with an elevated carcinoembryonic antigen level were computed tomography of the abdomen and pelvis (selected by 92\%), repeat carcinoembryonic antigen level (87\%), colonoscopy (87\%), chest X-ray $(71 \%)$, liver function tests $(69 \%)$ and complete blood count $(67 \%)$ (Table I). For the patient with suspected pulmonary metastases, computed tomography of the chest (90\%), computed tomography of the abdomen and pelvis (75\%), complete blood count (55\%), colonoscopy (55\%), liver function tests (55\%), repeat carcinoembryonic antigen level (53\%) and whole body positron emission tomography (utilizing ${ }^{18}$ F-fluorodeoxyglucose) scan $(51 \%)$ were selected by $>50 \%$ of the respondents. There were statistically significant differences in the patterns of diagnostic tests chosen between the two vignettes for carcinoembryonic antigen level, complete blood count, liver function tests, chest X-ray, colonoscopy, computed tomography of the abdomen and pelvis, magnetic resonance imaging of the abdomen and pelvis, computed tomography of the chest and positron emission tomography (utilizing ${ }^{18} \mathrm{~F}$-fluorodeoxyglucose) scan. Of these tests, carcinoembryonic antigen level, complete blood count, liver function tests, chest X-ray, colonoscopy and computed tomography of the abdomen and pelvis were recommended more frequently for the vignette featuring the patient with an elevated carcinoembryonic antigen level; only computed tomography of the chest and positron emission tomography (utilizing ${ }^{18} \mathrm{~F}$-fluorodeoxyglucose) scan were selected more frequently for patients with suspected pulmonary metastases. Few surgeons selected the option of recommending 'other' tests or procedures. Among the $10 \%$ of evaluable respondents who recommended other tests, there was a lack of congruence in the specific type of response. Physical examination, proctoscopy and endorectal ultrasonography were the most frequent 'other' tests chosen for the hypothetical patient with isolated elevation in carcinoembryonic antigen level. Abdominal ultrasonography, computed tomography-guided biopsy, needle biopsy and physical examination were the most frequent 'other' tests chosen for the hypothetical patient with suspected pulmonary metastases.

The $\chi^{2}$ analysis revealed that test use varied by physician age for selected tests. Due to small sample sizes for the $\leq 39$ and $\geq 60$ year age categories, they were collapsed in some cases into $\leq 49$ and $\geq 50$ year age categories, respectively, for statistical testing. This occurred for carcinoembry- 
Table I. Diagnostic workup patterns of members of the American Society of Colon and Rectal Surgeons for a patient with resected T3NOM0 rectal cancer and subsequent suspected recurrence.

\begin{tabular}{lcc}
\hline & \multicolumn{2}{c}{ Clinical scenario } \\
\cline { 2 - 3 } Testing modality & $\begin{array}{c}\text { Elevated carcinoembryonic } \\
\text { antigen level }\end{array}$ & Pulmonary nodules $^{\mathrm{a}}$ \\
\hline (Repeat) carcinoembryonic antigen level $^{\mathrm{b}}$ & 87 & 53 \\
Complete blood count $^{\mathrm{c}}$ & 67 & 55 \\
Liver function tests $^{\mathrm{b}}$ & 69 & 55 \\
Chest X-ray $^{\mathrm{b}}$ & 71 & 18 \\
Colonoscopy $^{\mathrm{b}}$ & 87 & 55 \\
Computed tomography of the abdomen/pelvis $^{\mathrm{b}}$ & 92 & 75 \\
Computed tomography of the chest $^{\mathrm{b}}$ & 44 & 90 \\
Magnetic resonance imaging of the abdomen/pelvis $^{\mathrm{d}}$ & 4 & 4 \\
Whole body positron emission tomography scan $^{\mathrm{c}}$ & 40 & 51 \\
(utilizing $^{18} \mathrm{~F}-$ fluorodeoxyglucose) & & 7 \\
Carcinoembryonic antigen scan $^{\mathrm{d}}$ & 8 & 7 \\
Bone scan $^{\mathrm{d}}$ & 5 & 7 \\
\hline
\end{tabular}

aThe numbers in these columns represent the percentage of respondents who use each test and are based on the total number of respondents $(\mathrm{n}=347)$. The $\chi^{2}$ analysis (or Fisher's, as appropriate) was used to compare use vs. non-use for each modality between the two types of recurrence. ${ }^{\mathrm{b}} \mathrm{p}<0.001,{ }^{\mathrm{c}} \mathrm{p}<0.01,{ }^{\mathrm{d}}$ not significant.

onic antigen level, colonoscopy, computed tomography of abdomen and pelvis, magnetic resonance imaging of the abdomen and pelvis, carcinoembryonic antigen scan and bone scan in the scenario describing the patient with an elevated carcinoembryonic antigen level. For the patient with suspected pulmonary metastases, computed tomography of the chest, magnetic resonance imaging of the abdomen and pelvis, carcinoembryonic antigen scan and bone scan, selection results were collapsed.

The diversity of tests selected for the hypothetical patient with an elevated carcinoembryonic antigen level differed significantly ( $\mathrm{p} \leq 0.05$, Fisher's and $\chi^{2}$ tests) by surgeon age only for complete blood count, liver function tests, chest X-ray and colonoscopy. Physicians in age categories 40-49 and 50-59 selected liver function tests and chest X-ray more frequently than those in the $\leq 39$ or $\geq 60$ years of age categories. Those $\leq 49$ years selected colonoscopy more frequently than those $\geq 50$. Tests selected for the hypothetical patient with suspected pulmonary metastases did not differ significantly (Fisher's and $\chi^{2}$ tests) by age.

Analysis of test use by US Census Region (Northeast, South, Midwest, West and non-US) for the patient with an elevated carcinoembryonic antigen level revealed significant differences (Fisher's and $\chi^{2}$ tests) among regions for positron emission tomography (utilizing ${ }^{18} \mathrm{~F}$-fluorodeoxyglucose) scan $(\mathrm{p}<0.001)$. Non-US respondents were significantly less likely to use positron emission tomography (utilizing ${ }^{18} \mathrm{~F}$-fluorodeoxyglucose) scan than US respondents. For the clinical scenario involving the patient with suspected pulmonary metastases, a significant difference $(\mathrm{p}<0.001)$ was found among regions for positron emission tomography (utilizing ${ }^{18} \mathrm{~F}$-fluorodeoxyglu- cose) and bone scan only. As in the elevated carcinoembryonic antigen scenario, non-US respondents were significantly less likely to use positron emission tomography (utilizing ${ }^{18} \mathrm{~F}$-fluorodeoxyglucose) scans than US respondents. For bone scan, however, non-US respondents were significantly more likely to use this test than US respondents. Overall, non-US respondents recommended the smallest variety of tests, utilizing 5 of the 11 tests offered on the menu. Analysis of test use by the health maintenance organization penetration rate $(\leq 30$ or $>30 \%$ ) revealed no significant differences among geographic sites in the US for either clinical scenario.

This analysis indicates that the clinical situation (in this case, elevated carcinoembryonic antigen level vs. suspected pulmonary metastases) led to large differences in the extentof-disease workup. Physician age was responsible for a modest amount of the measured variation. US Census Region had a statistically significant, but clinically small, effect on the recommended use of several diagnostic modalities employed in evaluating T3NOM0 rectal cancer patients with suspected cancer recurrence. Health maintenance organization penetration rate had no effect.

\section{Discussion}

Approximately $35 \%$ of patients with rectal carcinoma succumb to the disease within five years of diagnosis. Some have unresectable disease at initial diagnosis but many are treated initially with curative intent. Clinicians managing patients after curative intent treatment are highly motivated to detect recurrence promptly, anticipating that salvage therapy can be successful. Common sites of recurrence include liver, bone, 
lymph node, peritoneum and local recurrence. Guidelines for follow-up of asymptomatic rectal carcinoma patients are inconsistent $(10,11)$. There is little evidence supporting any particular workup strategy for patients with suspected recurrence. There are many testing and imaging modalities available, and the cost of intensive workup is potentially great. The present study represents the first empirical data from a large sample of experts who quantitatively described how they personally evaluate their own patients with clinically suspected recurrence. Analysis of practice patterns enables analysis of the economic consequences of medical decisions but does not discern the motivation underlying the decisions. Put differently, what physicians say they do may not match what they actually do in clinical practice. We did not measure actual practice in this study as the multiple sources of input into clinical decision-making are difficult and costly to quantify and analyze. We utilized a survey instrument to assess physician practice, since the clinical vignettes presented to the survey recipients were idealized to deliberately minimize the many factors that may affect physician practice. These include patient age, comorbid conditions, quality of insurance, personal financial status and personal treatment philosophies of both the physician and patient. Despite our deliberate deletion of these factors in each scenario, the data presented here show that there is a great deal of variability in the extent-of-disease investigation for patients with suspected recurrence. Computed tomography is utilized differently, depending on the type of recurrence, which is not surprising given that the scenario featuring pulmonary nodules suggests a different mechanism of metastasis than the scenario featuring a moderate rise in the serum carcinoembryonic antigen level. The small or absent effects of physician age, health maintenance organization penetration rate, and US Census Region on workup intensity may be due to factors such as continuing medical education. Our survey was carried out before positron emission tomography (utilizing ${ }^{18} \mathrm{~F}$-fluorodeoxyglucose)-computed tomography fusion imaging became widely available, which is likely to account for the low frequency of use of positron emission tomography (utilizing ${ }^{18} \mathrm{~F}$-fluorodeoxyglucose) imaging. This imaging modality is therefore likely to be underestimated in the current report. Nonetheless, our analysis provides the first evidence that surgeons of the American Society of Colon and Rectal Surgeons investigate their patients with clinical evidence of systemic recurrence in variable ways. The lack of consensus for most tests in current practice is likely due to multiple factors, including the patient's financial status, the quality of medical insurance held by patients in a particular surgeon's practice, physician training, patient expectations, practice setting and cultural factors; factors that our vignettes deliberately did not mention. There is little evidence documenting the value of any particular strategy as measured in terms of length of life extension or quality of life. We believe this is a significant cause of the observed variability.

Evidence-based practice guidelines for cancer patient follow-up after curative intent treatment are available for breast cancer and, to a limited extent, for colorectal carcinoma, but most are based on low quality evidence developed by informal consensus or by assessing the opinions of experts. This may be why guidelines have not been widely accepted.
Well-controlled prospective clinical trials of alternative forms of workup for patients with suspected recurrence of rectal carcinoma, while ideal in discerning the proper and most effective management, are probably impractical to carry out in clinical practice. Medical systems in wealthy societies are willing to pay approximately $\$ 50,000-100,000$ per qualityadjusted life year for medical care of various sorts (12-14). There is little evidence that a high-intensity workup strategy for rectal carcinoma patients who develop clinical evidence of recurrence is valuable, according to this criterion.

Overdiagnosis is clearly possible with a high-intensity workup strategy. Intensive workup also exposes patients to additional risks such as reactions to contrast agents and complications of procedures utilized to rule out cancer after false-positive tests. It is unknown whether the potential gain in survival justifies the cost, discomfort and emotional stress that such high-intensity strategies may cause for patients. Without well-publicized evidence from high-quality studies, uniformity in the extent-of-disease evaluation of patients with rectal carcinoma and clinically suspected recurrence will be difficult to achieve. This analysis indicates that the type of suspected recurrence accounts for a portion of the variability and physician strategies. The results of this analysis are likely to be of value to practitioners and insurers by showing the actual practice of highly credentialed experts, the best evidence we have in the absence of controlled trials.

The data presented here may also have value for medical personnel involved in a legal entanglement in which it is claimed that a 'standard of care' in a 'contemporary community practice' was not met. We have provided evidence that the workup after suspected recurrence of rectal cancer is quite variable. The only testing modality recommended by $\geq 90 \%$ respondents was computed tomography; even here, the clinical presentation (elevated serum carcinoembryonic antigen level vs. suspicious lung masses) led to differential test utilization. The data also provide a defense when so-called 'economic credentialing' in a health maintenance organization suggests that a physician is ordering tests excessively.

\section{References}

1. Jemal A, Siegel R, Ward E, et al: Cancer Statistics. CA Cancer J Clin 58: 71-96, 2008.

2. Johnson FE, Longo WE, Ode K, et al: Patient surveillance after curative-intent surgery for rectal cancer. Int J Oncol 27: 815-822, 2005.

3. Chassin MR and Galvin RW: National roundtable on health care quality: the urgent need to improve health care quality. JAMA 280: 1000-1005, 1998.

4. Kohn LT, Corrigan JM, Donaldson MS (eds): To Err is Human: Building a Safer Health Care System. National Academies Press, Washington, D.C., 1999.

5. Tsai DY, Virgo KS, Colberg JW, et al: The age of the urologist affects the postoperative care of prostate carcinoma patients. Cancer 86: 1314-1321, 1999.

6. Wennberg $\mathrm{J}$ and Gittlesohn A: Variation in medical care among small areas. Sci Am 246: 120-134, 1982.

7. Birkmeyer JD, Sharp SM, Finlayson SR, et al: Variation profiles of common surgical procedures. Surgery 124: 917-923, 1998.

8. Diehr P, Cain KC, Kreuter W and Rosenkranz S: Can small-area variation analysis detect variation in surgery rates? The power of small-area variation analysis. Med Care 30: 484-502, 1992.

9. Margenthaler JA, Fosko SW, Virgo KS, et al: Evaluation of patients with clinically suspected melanoma recurrence: Current practice patterns. Int J Oncol 21: 591-596, 2002. 
10. Renehan AG, Egger M, Saunders M and O'Dwyer ST: Impact on survival of intensive follow-up after curative resection of colorectal cancer: systemic review and meta-analysis of radomised trials. BMJ 324: 1-8, 2002.

11. Johnson FE and Virgo KS (eds): Cancer Patient Follow-up. Mosby, St. Louis, 1997.

12. Earle CC, Chapman RH, Baker CS, et al: Systematic overview of cost-utility assessments in oncology. J Clin Oncol 18: 3302-3317, 2000.
13. Cormier JN, Xing Y, Ding M, et al: Cost-effectiveness of adjuvant interferon in node-positive melanoma. J Clin Oncol 25: 2442-2448, 2007.

14. Van den Hout WB, Peul WC, Koes BW, et al: Prolonged conservative care versus early surgery in patients with sciatica from lumbar disc herniation: cost utility analysis alongside a randomised controlled trial. BMJ 336: 1351-1354, 2008. 